\title{
Un exemple de pluralisme juridique : l'accord "grands routiers" du
} 23 novembre 1994

\section{Stéphane Carre}

\section{Citer ce document / Cite this document :}

Carre Stéphane. Un exemple de pluralisme juridique : l'accord "grands routiers" du 23 novembre 1994. In: Revue juridique de l'Ouest, 1999-3. pp. 377-394;

doi : https://doi.org/10.3406/juro.1999.2533

https://www.persee.fr/doc/juro_0990-1027_1999_num_12_3_2533

Fichier pdf généré le 05/05/2018 


\title{
UN EXEMPLE DE PLURALISME JURIDIQUE :
}

\section{L'ACCORD "GRANDS ROUTIERS" DU 23 NOVEMBRE 1994"}

\author{
par \\ Stéphane CARRE \\ Maître de conférence à l'IUT de Saint-Nazaire, Université de Nantes \\ UPRES-A CNRS 6028/CERL
}

La concurrence exacerbée entre entreprises et les conditions de travail de plus en plus difficiles dans le secteur du transport routier de marchandises ont abouti à des conflits sociaux particulièrement durs à partir de $1989^{2}$. La mise en place du permis à points fut le détonateur qui mis brutalement en évidence les tensions existantes. Chauffeurs artisans et salariés, ne respectant qu'occasionnellement la législation, considérèrent leur emploi directement menacé par la nouvclle lćgislation ${ }^{3}$.

Mais comment assainir une profession qui ne respecte traditionnellement pas la législation qui lui est applicable? La solution adoptée fut celle d'associer le plus étroitement possible les organisations professionnelles et syndicales à la définition des mesures nécessaires. Le procédé a pour avantage de faciliter la recherche d'un consensus entre les différents intérêts en présence. Il a cependant pour limite de présupposer l'existence d'une réelle représentativité des organisations professionnelles, alors même qu'il s'agit d'un secteur d'activité particulièrement hétérogène.

Ainsi va naître le "contrat de progrès" (mars 1994), ensemble de propositions d'actions faites par un groupe de travail réunissant des représentants

1 - Cet article a fait l'objet d'une communication lors des "deuxièmes journées d'échanges et de recherche en logistique et transport", les 26 et 27 novembre 1998 à l'I.U.T. de Saint-Nazaire.

2 - Longue suite de conflits sociaux : juillet 1989, juillet 1992, automne 1996, automne 1997. Mais d'autres conflits ont précédemment eu lieu : 1971, 1973, 1984.

3 - Le principe du permis à points fut institué dès 1989 (loi n89-469 du 10-7-1989). Mais sa mise en place effective n'intervint qu'en 1992, entraînant alors un grave conflit. 
de l'administration, des personnalités qualifiées et les partenaires sociaux. Parmi les propositions retenues, il fut convenu que, en matière de temps de travail, la voie choisie serait celle d'une convention collective. Le 23 novembre 1994 était conclu "l'accord sur le temps de service, les repos récupérateurs et la rémunération des personnels de conduite marchandises grands routiers ou longue distance".

Or, cet accord, censé clarifier et améliorer la situation juridique des conducteurs routiers, reproduit l'une des caractéristiques essentielles de l'état du droit antérieurement en vigueur. En particulier, les règles que pose cet accord sont parfois contraires à la législation d'ordre public préexistante : de même que les pratiques antérieures tendaient à nier la présence d'une réglementation étatique, l'accord de 1994 comporte des règles illégales. Il semble donc ne pas toujours prendre en compte cette réglementation (1).

Mais ces règles illégales ne sortent-elles pas dès cet instant de l'ordonnancement juridique? Rien ne semble plus évident. Rien n'est pourtant moins sûr.

Ces dispositions quittent certes l'ordre juridique garanti par les tribunaux de l'F́tat. Mais ces règles semblent pouvoir trouver ailleurs que devant ces juridictions les moyens de leur application. Ainsi se met se place un ordre juridique parallèle à celui de l'État (2).

\section{I - LE COUP DE FORCE DE L'ACCORD DU 23 NOVEMBRE 1994}

L'accord sur les temps de service des personnels "grands routiers" est une convention collective largement dérogatoire aux dispositions légales concernant les temps de travail. Il est vrai que de telles conventions sont aujourd'hui possibles, sur la base des articles L. 132-26 et L. 212-2 du Code du travail. Cependant, la validité de tels accords est soumise à certaines conditions de fond. La convention collective ne peut déroger à tout.

Selon l'article L. 212-2 alinéa 3 du Code du travail, une convention collective étendue peut seulement déroger aux décrets d'application concernant l'aménagement de la durée légale du travail pour leurs dispositions relatives à "l'aménagement et à la répartition des horaires de travail à l'intérieur de la 
semaine, aux périodes de repos, aux conditions de recours aux astreintes, ainsi qu'aux modalités de récupérations des heures de travail perdues".

Or, l'accord du 23-11-1994 stipule notamment que, à compter du 1-101995 et jusqu'au 31-12-1996, puis à partir du 1-1-1997 et jusqu'au 31-12-1997, les temps de service ne pourront être supérieurs, respectivement, à 60 heures et 56 heures par semaine (art.VII.1 de l'accord) ${ }^{4}$. Ces durées limites sont évidemment supérieures à celles prévues au décret $n^{\circ} 83-40$ du 26-1-1983 relatif à la durée du travail dans les entreprises de transport routier, qui fixe la durée maximale de service à 52 heures sur une semaine (art. $5 \S 4$ ).

Ces dispositions conventionnelles dérogatoires aux décrets d'application concernant l'aménagement du temps de travail sont donc illégales en ce que la loi n'a jamais autorisé par voie conventionnelle un dépassement de la durée maximale du travail ou de service.

Par ailleurs, l'accord de novembre 1994 établit un mode de calcul de la durée du travail sur le mois et non sur la semaine (art. IV.2). Or les accords dérogatoires ne doivent pas moins maintenir un mode de calcul sur la semaine. En cas d'annualisation du temps de travail ou d'accord de cycle, c'est la durée moyenne de travail sur une semaine qui doit rester en dernière analyse le point de référence. Cette disposition semble donc être contraire tant à l'article L. 212-2 du Code du travail qu'à l'article L. 212-5 du Code du travail.

Au surplus, il aurait été nécessaire que ces dispositions fassent l'objet d'un arrêté d'extension pour être valides puisque seule une convention collective étendue peut comporter des dispositions dérogatoires à certaines questions touchant à l'aménagement du temps de travail et des horaires (L. 212-2 C. trav.). Or, il n'a jamais été procédé à la procédure d'extension ${ }^{5}$.

D'un point de vue normatif, ces règles illégales doivent être écartées. De même, la licéité des accords d'entreprise conclus en application des dispositions illégales de l'accord "grands routiers" peut être contestée. Certaines

4 - Au-delà de l'année 1997, les partenaires sociaux disposent de nouvelles réductions des temps de services, sous la condition d'une harmonisation des règles sociales à l'ensemble des entreprises de transports routiers de l'union européenne (art. VII.2 c de l'accord).

5 - Pour une démonstration détaillée du caractère illégal de cet accord, cf. M. Hautdidier et P. Ramackers: "La durée du travail est-elle encore contrôlable. Réflexions sur une évolution à partir de l'exemple des transports routiers" D.S. avril 1995, p. 335 et s. 
décisions judiciaires semblent d'ailleurs aller dans ce sens, qui ont abouti à la condamnation de transporteurs ayant partiellement suivi l'accord "grand routiers", sur le fondement du non respect de la législation du travail ${ }^{6}$. Mais surtout, la chambre criminelle de la Cour de cassation a jugé qu'un employeur ne pouvait être sanctionné dès lors, qu'appliquant l'accord "grands routiers", il ne respectait pas la législation du travail'. La Cour de cassation considère effectivement que l'employeur commet une "erreur de droit", excusant le non respect du Code du travail, en appliquant un accord illicite, mais communiqué par l'administration, par ailleurs présente lors des négociations préalables à la signature.

Mais la réalité est autrement plus complexe. Peut-on rejeter dans un "néant juridique" des règles contraires à la loi lorsque la réglementation, par ses caractéristiques et les modalités de sa mise en œuvre, ne favorise en rien une stricte application?

Car, on le sait, la législation étatique en matière de durée du travail dans le transport routier, est déjà largement dérogatoire au droit commun. Ces dérogations sont censées rapprocher le droit des "réalités du terrain". Mais ce faisant, ce sont les pratiques qui imposent leur présence au droit et non l'inverse. Il est dès lors présomptueux d'espérer voir une évolution rapide des comportements sous l'influence de la loi. D'autre part, l'application de cette réglementation est restée longtemps sans véritable contrôle, de nombreuses dispositions ne faisant au demeurant l'objet d'aucune sanction dissuasive en cas d'irrespect ${ }^{8}$.

Aussi, les temps de service maxima fixés par l'accord de 1994 correspondent grosso modo à ceux existant en pratique. Ainsi, sur le mois, les temps de service d'un chauffeur routier "longue distance" étaient de 250 heures, au début des années quatre-vingt-dix ${ }^{9}$. L'accord de 1994 stipule un temps de service maximal égal à 240 heures par mois, pour l'année 1996. Les pratiques que

6 - cf. trib. de police de Pithivier, 26-2-1998 BTL nº 2743 9-3-1998, p. 171 et s.- et BTL ${ }^{\circ} 2744,16-3-$ 1998, p. 201.

7 - Crim. 24.11.1998 B.T.L. ${ }^{\circ} 2783,18-1-1999$, p. 18.

8 - Sur tous ces points, largement connus, cf. entre autres : Rapport Dobias "La situation économique et sociale du transport routier de marchandises" janvier 1993, annexe 3 : Note de la direction de la prévision du ministère de l'économie et des finances sur les transports routiers de marchandises 1992, p. 6, annexe 8 : Note du ministère de l'équipement, du logement et des transports 1992, p. 2 ; BTL $\mathrm{n}^{\circ} 2592$ 12-12-1994, p. 883 ; BTL n 2717 , 1-9-1997, p. 560 ; F. Ocqueteau, J.C. Thoenig : "Mouvements sociaux et action publique : le transport routier de marchandises" Sociologie du travail $n^{\circ} 4 / 97$, p. 397, 407)...

9 - Sur la base de 62,5 H./sem. (BTL n 2608 -10-4-1995, p. 252) x 4 semaines. 
la réglementation a implicitement autorisées trouvent ainsi leur consécration en prenant une consistance juridique.

Les partenaires sociaux l'ont d'ailleurs voulu ainsi. Il s'agissait d'abord d'établir les conditions d'une transparence des durées des temps de service et de leur rémunération puis de prévoir une réduction programmée de celles-ci ${ }^{10}$. Il fallait donc partir des pratiques telles qu'elles existaient et les officialiser ${ }^{11}$.

Les partenaires sociaux ont parallèlement cherché à exclure l'application de la législation étatique. Par exemple, une note de l'Union des Fédérations de Transport du 8-3-1995 prône une application distributive, et non pas cumulative, de l'accord du 23-11-1994 et du décret du 26-1-1983. Aux entreprises assujetties à cet accord qui ne le respecteraient pas, sera appliquée la réglementation nationale en vigueur (point $\mathrm{I}-1)^{12}$. Il peut en être déduit que les employeurs qui mettront en œuvre l'accord "grand routier" seront dispensés d'appliquer par ailleurs la législation étatique en matière de durée et d'aménagement du temps de travail. Or, nonobstant les dispositions dérogatoires de l'accord de 1994, il est étonnant de voir mettre ainsi entre parenthèses cette réglementation.

Dira-t-on de cette nouvelle métamorphose de l'état du droit dans le secteur des transports routiers de marchandises qu'elle représente l'apparition d'un phénomène d'infra-droit? Il nous semble que la transformation est plus profonde.

Il est difficile de penser l'infra-droit autrement que comme un phénomène "sous le droit". Il y aurait donc d'une part le droit et d'autre part des manifestations de la réalité qui prendraient les formes d'un phénomène juridique, sans en être. L'infra-droit serait encore des manifestations du phénomène juridique, mais qui resteraient étrangères au droit positif, en tant que telles ${ }^{13}$.

10 - Cf. les considérants introductifs de l'accord du 23-11-1994.

11 - Des précédents existent. Ainsi, selon $M$. Bonnechère, le "protocole" sur la flexibilité, conclu en 1984, n'était rien d'autre qu'unenégociation sur la transgression de la réglementation ("Les tendances à la déréglementation et leur incidence sur la théorie des sources en droit du travail" D.S. janvier 1990, p. 42). 12 - L'U.F.T. regroupe un certain nombre d'organisations patronales signataires de l'accord de novembre 1994. Cette interprétation correspond aux souhaits émis dès le rapport Dobias, notamment par la F.N.T.R. Cette organisation patronale prônait une amélioration des conditions de travail des conducteurs routiers "principalement par voie conventionnelle et non par voie réglementaire non concertée" (rapport Dobias ; annexe 13, p. 5).

13 - Infra-droit : "tout ce qui, n'étant pas droit au sens strict (droit étatique ou régulation reconnue comme droit par l'État, participe néanmoins du phénomène juridique lato sensu" (A.J. Arnaud) 
Dans une perspective normativiste, toute règle illégale peut donc être perçue comme constituant un infra-droit parce qu'elle se trouve exclue d'un ordre juridique qui se veut unitaire.

Cependant, force est de constater que les dispositions illégales de l'accord de 1994 ne peuvent être perçues comme ne constituant que les apparences d'un phénomène juridique. En réalité, toutes les caractéristiques convergent pour faire de cet accord un phénomène juridique à part entière. Tout à la fois dans et hors le droit, il est un exemple éclatant de pluralisme juridique.

\section{II - L'IDENTIFICATION DES DISPOSITIONS ILLEGALES DE L'ACCORD A DU DROIT}

Les phénomènes de pluralisme juridique peuvent parfaitement s'accommoder d'un système juridique unitaire. Tout dépend de l'extension que l'on donne à cette notion. De la sorte, le seul fait que des organisations ou des groupements particuliers (associations, syndicats, administrations...) se dotent de règles internes ou conventionnelles spécifiques (conventions collectives, statuts, règlements intérieurs, circulaires, techniques contractuelles ad hoc...) peut être perçu comme une manifestation de pluralisme juridique parce que, même si elles ne sont pas contraires à des règles impératives, elles contribuent à une diversité du droit que l'analyse restreinte à la seule législation nous cache. C'est du reste ainsi que G. Gurvitch entendait d'abord le pluralisme juridique ${ }^{14}$.

Un auteur tel que G. Teuber perçoit un phénomène de pluralisme juridique dans le fait que des discours ou des logiques différentes et antagonistes soient à l'œuvre au sein du système juridique. L'ordre juridique incorpore ces diverses logiques (économique, notamment) en en faisant la traduction juridique et permet ainsi la confrontation permanente de ces discours ${ }^{15}$.

Mais la difficulté devient grande d'admettre un phénomène de pluralisme juridique quand des règles normatives tout à fait originales

Dictionnaire encyclopédique de théorie et de sociologie du droit LGDJ Paris 1993 (2ème éd.) ; cf. également J. Carbonnier : "Sociologie juridique" Paris PUF Thémis 1978, p. 218 et s.

14 - cf. notamment G. Gurvitch :"L'idée de droit social" 1972 Scienta Verlag Aalen éd. (1 ère éd. : Paris Sirey 1932), p. 39 et s.- : "Problèmes de sociologie du droit" Traité de sociologie (sous la dir. de G. Gurvitch\} II 1968 PUF Paris -spéc. p. 190 et s.).

15 - Cf. G. Teubner : "Altera pars audiatur : le droit dans la collision des discours" Droit et Société $\mathrm{n}^{\circ}$ 351997 , p. 99 et s.-. 
apparaissent, qui heurtent de front un ordonnancement juridique unitaire. A priori, aucune greffe ne peut prendre. Il y aura un rejet des règles illicites hors de l'ordre juridique officiel, dont l'Etat est garant ${ }^{16}$.

Cependant, cette position ne fait pas cas de la question de la juridicité d'une règle. Hors ce problème est distinct de celui de celui de la validité d'une norme juridique au regard d'un système juridique particulier. De cette façon, nous savons qu'une règle de droit étrangère ne peut pas être mise en œuvre par nos tribunaux lorsqu'elle est contraire à l'ordre public français. Mais cela ne signifie évidemment pas que cette règle n'a aucune consistance juridique par ailleurs. Quels sont les critères qui font d'une norme quelconque une norme juridique? Tel est le problème de la juridicité d'une règle ${ }^{17}$.

Les dispositions illégales de l'accord du 23-11-1994 émergent à la vie juridique selon deux voies. D'une part, l'accord organise lui-même les moyens de son application et réinvente aisément les techniques qui font d'une règle une norme juridique (A). D'autre part, l'État est intervenu afin de soutenir l'application de cette convention. L'accord se trouve dès lors impliqué dans les mécanismes par lesquels l'État entend faire appliquer les règles de droit qu'il pose ou qu'il reconnaît (B).

\section{A - Les propriétés juridiques inhérentes à l'accord}

L'accord de novembre 1994 se présente sans ambiguïté aucune comme un accord engageant différentes parties, et plus précisément comme une convention collective du travail. Les formes et les dispositions de cet accord sont typiques des engagements contractuels, dont l'objectif est de s'obliger mutuellement. En particulier, l'accord fixe un certain nombre d'obligations précises à destination des employeurs ${ }^{18}$. Il semble que les dispositions de la

16 - Il existe une abondante littérature sur la question du pluralisme juridique. En langue française, outre les auteurs déjà cités, on peut se référer à quelques ouvrages classiques (J. Cruet : "La vie du droit et l'impuissance des lois" Flammarion 1908 ; M. Hauriou : "Théorie de l'institution et de la fondation" Paris 1925 ; M. Weber : "Sociologie du droit", Paris PUF 1986 ; J. Carbonnier : "Flexible droit" Paris LGDJ 1983 ; Santi Romano : "L'ordre juridique" Paris éd. Dalloz 1975) ainsi qu'à la thèse de J.G. Belley : "Conflit social et pluralisme juridique en sociologie du droit" 1977 Paris II.

17 - Sur cette question, cf. notamment : D. de Béchillon : "Qu'est-ce qu'une règle de droit ?" Paris éd. $O$. Jacob 1998, p. 131 et s., p. 147 et s., p. 230 et s. ; L. Assier-Andrieu : "Le droit dans les sociétés humaines" Paris éd. Nathan 1996, principalement la première partie.

18 - Dispositions codifiées et précisément classées (plan d'ensemble, articles...), liste et signatures des parties contractantes, dates de signature et d'entrée en vigueur, lieu de signature, publicité de l'accord par 
convention conformes à la réglementation de l'État puissent trouver à s'appliquer devant les tribunaux ${ }^{19}$. Mais quel caractère contraignant pour les dispositions qui ne sont pas conformes à la loi ?

L'accord de novembre 1994 évoque parfois des sanctions permettant la répression de certains agissements contraires à la convention. L'accord vise en particulier les mauvaises manipulations du chronotachygraphe, dont l'utilisation afin de décompter les heures de service des chauffeurs est obligatoire (art.III.2-lde l'accord $)^{20}$. Dans la mesure où ces règles ne concernent pas directement les dispositions illicites de la convention, elles peuvent être invoquées sans difficultés devant les tribunaux de l'État.

De même, rien ne paraît s'opposer à ce que les différents mécanismes d'incitation à l'application de l'accord "grand routiers" ne puissent être invoqués devant un tribunal d'État, dès lors qu'ils ne sont pas contraires à la loi (cf. infra). De façon plus général, l'obtention d'une réparation sur le fondement d'une disposition conventionnelle paraît toujours possible dès l'instant que cette disposition n'est pas illicite ${ }^{21}$.

Mais ces règles secondent efficacement la mise en œuvre des dispositions illégales en incitant au décompte précis des heures de services, y compris quand elles dépassent la durée maximale du travail.

dépôt à la Direction départementale du travail et au secrétariat greffe du Conseil de prud'hommes de Paris... Il reste ici que l'effet relatif du contrat ne rendrait obligatoire l'exécution d'une telle convention qu'entre les signataires directs.

19 - Cf., par exemple, J. Chotard : "Durée du travail et salaire dans les transports de marchandises par route" D.O. avril 1997 -p157 et s. Il faut cependant noter que l'article L. 212-2 . du Code du travail, en subordonnant la validité des dispositions dérogatoires à l'existence d'un arrêté d'extension touchant l'accord, paraît remettre en question la validité de l'ensemble de la convention en l'absence d'un tel arrêté. D'ailleurs, le ministère aurait pu prendre un arrêté d'extension concernant l'accord du 23 novembre 1994, après en avoir écarté les dispositions illicites (L. 133-8 C. trav.).

20 - Il s'agit de sanctions disciplinaires si le salarié en est responsable ou des sanctions prévues par la réglementation si les mauvaises manipulations sont du fait de l'employeur (art. III.2 -I- de l'accord). L'accord fait cependant référence à des dispositions réglementaires imaginaires puisque, en 1994, il n'était pas encore prévu à la législation de sanctions pénales en cas de falsification des disques du chronotachygraphe.

21 - De plus, concernant les durées de service supérieures à la durée maximale du travail autorisée par la loi, l'employeur ne peut se prévaloir, devant les tribunaux, de leur caractère illégal pour ne pas les rémunérer. Toute période travaillée doit être effectivement rémunérée. A l'inverse, un salarié va toujours pouvoir réclamer le paiement de ces heures, dès lors qu'elles ont été effectivement travaillées. 
La constatation est d'importance. L'intégration de règles illégales au sein d'une "construction juridique" par ailleurs licite facilite la mise en œuvre des dispositions contestables. Le critère de légalité vient ainsi au secours du critère de juridicité en permettant de peser indirectement en faveur de l'application de ces dispositions.

Mais il nous semble que le caractère juridique des dispositions illégales résulte d'abord des moyens pouvant être mis en œuvre afin d'inciter directement les parties à respecter leurs obligations. De ce point de vue, beaucoup s'accordent à dire que la juridicité d'une norme dépend de l'existence d'une sanction socialement organisée, c'est-à-dire de procédures permettant la constatation de l'irrespect d'une règle et la mise en œuvre éventuelle d'une sanction $^{22}$. La figure du juge vient alors immédiatement à l'esprit ${ }^{23}$.

Or, le recours aux juridictions d'État ou même à un arbitre ne nous semble pas être les seuls instruments possibles d'une sanction socialement organisée. L'intervention d'une tierce personne peut prendre des formes moins "sévères" que celles consistant à trancher un litige puis d'avoir recours, le cas échéant, à la contrainte sociale garantie par l'État, si besoin par une exécution forcéc. Ce que souhaitent d'ailleurs les partenaires sociaux, c'est une application globale de l'accord par des mécanismes d'incitation.

Cette incitation découle de plusieurs mécanismes. La convention stipule d'abord qu'un employeur pourra engager des négociations afin de conclure un accord dans l'entreprise (art.III.2-III de l'accord). L'accord recherché est une véritable convention collective et non un accord atypique : seuls les

22 - Encore faut-il souligner que la présence de sanctions socialement organisées est un critère fort critiqué de la juridicité d'une norme. Cf. , par exemple, A. Jeammaud : "La règle de droit comme modèle" chon. XXXIV D. 1990, p. 207 à 209-, "Consécration de droits nouveaux et droit positif : sens et objet d'une interrogation" in "Consécration et usages de droit nouveau" (1985) CERCRID Univ. de Saint Etienne -p. 12 et s.- ; P. Amselek : "L'évolution générale de la technique juridique dans les sociétés occidentales" R.D.P. 1982, p. 275 et s. ; D. de Béchillon : "Qu'est-ce qu'une règle de droit ?" Paris éd. O. Jacob 1998, p. 59 et s., p. 250 et s.

23 - Il semble aussi que la seule présence de cette tierce personne, en l'absence même de règles juridiques préalables, puisse être le signe distinctif d'un phénomène juridique. Cf., parmi bien des auteurs, $M$. Weber, dans ses propos relatifs aux formes originaires du droit, le plus généralement de nature "irrationnelle" et "matérielle" ("Sociologie du droit", PUF Paris 1986, spéc. p. 32, 39 et s., p. 119 et s...). 
employeurs ayant dans leur entreprise une délégation syndicale pourront entamer une telle négociation. L'accord est transmis à l'inspection du travail ${ }^{24}$.

A défaut d'accord dans l'entreprise, la convention de novembre 1994 invite ensuite l'employeur à se rapprocher de l'inspection du travail afin de lui présenter les initiatives qu'il compte prendre pour la mise en cuvre effective de l'accord "grands routiers" (art. III.2 -IV) ${ }^{25}$. Enfin, le contrat de travail des conducteurs concernés doit faire référence à l'article III.2 de l'accord.

Nous sommes en face de différents relais visant à engager plus avant les partenaires sociaux dans l'application de l'accord du 23-11-1994. Mais comme l'accord lui-même, ces incitations résultent principalement d'un engagement public et officiel. A défaut de l'existence d'une tierce personne dont la fonction serait de peser pour le moins sur l'application de la règle, est-on en présence d'une véritable norme juridique?

Nous allons voir au demeurant que cette tierce personne existe. Néanmoins, il est bon de s'interroger sur le caractère juridique d'une règle indépendamment de la présence clairement identifiée de cette tierce personne.

A cet égard, l'existence d'un engagement précis et public peut-il être perçu comme un simple engagement moral? Il n'en est pas certain. En effet, cet engagement se trouve officialisé par rapport à des tiers non directement intéressés au respect des obligations affichées. L'engagement moral, présent dans tout contrat, devient aussi un engagement social ${ }^{26}$. Enfin, la formalisation dont fait

24 - Les dispositions de l'accord évite soigneusement d'indiquer que les conventions d'entreprise fixeront les temps de service, y compris ceux se trouvant au dessus des maxima légaux. Ces conventions ou les initiatives patronales communiquées à l'inspection concernent simplement le décompte des heures, leur rémunération, ainsi que la "diminution effective des temps de service". Mais la légalité du recours à des accords d'entreprise a pu être contestée : M. Hautdidier et P. Ramackers : "La durée du travail est-elle encore contrôlable? Réflexions sur une évolution à partir de l'exemple des transports routiers" D.S. avril 1995 , p. 336.

25 - Un délai est prescrit avant la fin duquel l'employeur doit avoir entamé ces démarches. L'avis de l'inspection du travail est demandé. Cet avis est communiqué au personnel. A défaut de réponse de l'inspection, l'employeur engage ces initiatives et en communique les résultats à l'inspection. Toute cette procédure est rappelée dans la "circulaire" d'application U.F.T. du 8-3-1995 (point II.2.2 b).

26 - Cf. les analyses pertinentes de G. de Tarde ("Les transformations du droit" éd. Berg. International 1994, spéc. p. 111 et s.). 
l'objet la convention n'a-t-il justement pas pour objectif d'assurer précisément le respect de l'obligation par delà le seul engagement moral $^{27}$ ?

Une règle précise est posée. Elle s'extériorise et prend une vie propre, indépendamment du respect de la parole donnée, qui est une simple vertu. Mais la formalisation dont fait l'objet cette norme semble instaurer plus qu'une règle sociale, simple habitude ou pression exercée par le milieu. Bref, la juridicité d'un phénomène ne doit-il pas être parfois recherchée, non en fonction d'une sanction socialement organisée, mais à partir de l'existence d'un engagement socialement organisé ?

La précision des engagements, leur caractère technique et public semblent en tout cas appeler un contrôle institué et externe de l'application des normes fixées (examen détaillé des engagements, analyse concomitante des faits. enquête contradictoire...). Un simple contrôle social et informel ne saurait en l'occurrence suffire. Par contre, nous pouvons poser l'hypothèse que la simple existence d'organes de contre-pouvoir, telle une presse indépendante, peut assurer de fait ce contrôle extérieur.

Mais, quoi qu'il en soil, l'accord "grands routiers" stipule qu'il "est crée une commission nationale de suivi de l'accord entre les parties signataires au sein de la commission nationale d'interprétation et de conciliation" (art. VIII.1 $)^{28}$. Cette commission a pour mission de s'assurer du respect des dispositions de l'accord, d'examiner les éventuels litiges, de faire un bilan régulier de l'application de l'accord. Il est donc mis en place un organe tiers, chargé de peser sur l'application de la règle. Un critère déterminant de la juridicité de ce texte, et particulièrement des dispositions non conformes à la loi, existe donc.

\section{B - Le concours de l'État}

Cependant, la présence de la commission de suivi n'est peut-être pas l'aspect le plus original des moyens mis en place pour favoriser l'application de l'accord du 23-11-1994. En effet, l'État, cherche à inciter directement à l'application d'une convention dont il sait qu'elle comporte des règles illćgalcs. Il

27 - Par exemple, une publicité sous la forme d'une publication, d'un d'affichage, d'une proclamation, d'un dépôt, mais encore l'existence d'une codification détaillant et clarifiant les différentes obligations. 28 - Au demeurant, une telle disposition est très courante dans les conventions collectives de branche. 
est vrai que l'État ne peut se désintéresser de la mise en œuvre de l'accord : à défaut d'être partie à la convention, il a été partie prenante à la négociation ${ }^{29}$.

Certes, l'Etat n'a pas pris d'arrêté d'extension concernant l'accord "grands routiers", compte tenu probablement du caractère illicite de certaines de ses dispositions. Mais il n'a pas tenté non plus d'empêcher l'application de l'accord en demandant au ministère public d'invoquer d'office une exception de nullité, lors d'un litige prud'homal, par exemple ${ }^{30}$. Au contraire, il a été demandé aux fonctionnaires de l'Etat de s'engager en faveur de la mise en ouvre de l'accord.

Nous avons déjà souligné que l'accord "grands routiers" invitait les partenaires sociaux à se rapprocher de l'inspection du travail. Mais l'État luimême considère-t-il pertinente la démarche inverse, celle d'exiger des services de l'inspection du travail qu'ils favorisent la mise en cuvre de l'accord de 1994? La réponse est assurément positive, au grand dam d'inspecteurs et de contrôleurs du travail, plus habitués à faire respecter la législation du travail que des dispositions illicites ${ }^{31}$.

La circulaire $\mathrm{n}^{\circ}$ 94-94 du 29-12-1994 indique que l'objectif d'une diminution progressive des durées de service des conducteurs "grands routiers" devra être mis en ceuvre dans chaque entreprise concernée en liaison avec l'inspection du travail des transports. Les seuils fixés par l'accord, en matière de durée maximale de services sont considérés comme des "butoirs extrêmes" ne devant faire l'objet d'aucune "tolérance" ni "servir de prétexte à des errements" (point II-B-2 de la circulaire).

La circulaire invite les fonctionnaires de l'inspection du travail des transports à développer les contacts nécessaires avec les entreprises afin que les modalités d'application envisageables de l'accord de 1994 soient déterminées "ensemble" par le chef d'entreprise et les représentants de l'État (point III-1). La

29 - L'État a été partie prenante lors des travaux ayant successivement abouti au rapport Dobias et au "Contrat de progrès" (Commissariat général du plan) puis lors des négociations ayant précédées l'accord du 23.11.1994 (Cf. les propos liminaires de la circulaire $\mathrm{n}^{\circ}$ 94-94 du 29.12.1994).

30 - Sur cette question, cf. G. Lyon-Caen : "Négociation collective et législation d'ordre public" D.S. 1973 , p. 90.

31 - Cf. les propos de M. Hautdidier et P. Ramackers : "La durée du travail est-elle encore contrôlable ?" D.S. avril 1995, p. 333 et s. 
circulaire appelle aussi les fonctionnaires à développer une véritable fonction de conseil et d'encouragement (point III-2).

Elle préconise encore la mise en place dans chaque région, à l'initiative du directeur régional du travail des transports, d'une instance locale de suivi, composée de représentants de la profession et d'organisations syndicales, le directeur régional ou son représentant étant présent lors des réunions. Ces instances locales de suivi sont évidemment en relation avec la commission nationale de suivi. Ces instances doivent permettre de faire pression sur les entreprises pour qu'elles appliquent effectivement les dispositions conventionnelles : elles doivent faire en sorte que "l'engagement des parties signataires soit véritablement relayé dans les entreprises au plan local" (point III-2 de la circulaire).

L'État va par ailleurs progressivement modifier la législation en vigueur afin de rendre plausible l'application de l'accord de 1994. Les nombreuses réformes législatives intervenues depuis cette date ont manifestement cet objectif. L'accord "grands routiers" a donc un effet dynamique sur la réglementation. L'État tente ainsi de réduire l'écart entre les obligations de ceux qui ont dû s'engager dans l'application de l'accord et ceux qui échappent à sa mise en œuvre ${ }^{32}$. Il va s'agir encore d'inscrire dans la réglementation les moyens originellement fixés par l'accord et grâce auxquels peuvent être précisément calculé les temps de service ${ }^{33}$.

Mais l'État va aussi intervenir de façon beaucoup plus directe au regard de l'accord de 1994. La circulaire dispose que les employeurs qui s'inscriront "loyalement" dans le dispositif conventionnel devront être encouragés

32 - Du fait que l'accord de 1994 n'a pas fait l'objet d'une procédure d'extension, les employeurs qui ne sont pas adhérents à un groupement patronal signataire de l'accord n'ont pas à l'appliquer. Ne sont pas non plus concernés par l'accord, les artisans n'ayant pas de personnel salarié. Cependant, les nombreuses réformes intervenues viennent donc renforcer les obligations légales de ces entreprises, en particulier en jouant sur le volet commercial de l'activité de transporteur ou sur l'aspect de la sécurité routière : cf. notamment, depuis l'accord paritaire de 1994, la loi $\mathrm{n}^{\circ}$ 95-96 du 1-2-1995, le Décret $\mathrm{n}^{\circ}$ 95-541 du 2-51995, le Décret $n^{\circ}$ 95-601 du 5-5-1995, la loi $n^{\circ} 96-603$ du 5-7-1996, le Décret du 6.11.1996, la loi ${ }^{\circ}$ 98-69 du 6.2. 1998. La circulaire d'application $n^{\circ} 98-24$ du 18.2.1998 relative à l'application de la loi du 6.2.1998 rapelle au demeurant, dans son préambule, qu'il appartient à l'Etat "d'être le garant des accords conclus entre représentants des entreprises et des salariés et de faire respecter l'ensemble des règles économiques et sociales".

33 - D. $\mathrm{n}^{\circ}$ 96-1082 du 12-12-1996, art. 3-2 (mise en œuvre du chronotachygraphe et modalités de décomptes). 
le cas échéant par une aide des pouvoirs publics en matière de formation, de prévention des accidents du travail et plus généralement d'organisation du travail et de modernisation de leur gestion (point III-2).

Par un décret du 12-5-1997, L'État incite effectivement à la mise en œuvre de l'accord "grands routiers" en accordant une réduction des charges sociales aux entreprises qui appliquent la convention ${ }^{34}$. Cette aide précise apportée par l'État renforce incontestablement la juridicité des dispositions illégales de l'accord. Elle permet, dans une certaine mesure, la réintégration de ces dispositions dans l'ordonnancement juridique (la limite étant que ces règles n'en restent pas moins illégales). Elle suppose surtout l'existence d'un véritable contrôle de l'État et donc que s'exerce une pression socialement organisée.

L'État invite de plus ses fonctionnaires à prendre des mesures répressives à l'encontre des employeurs récalcitrants. Les corps de contrôle de l'État qui viendraient à avoir connaissance de pratiques inacceptables ou qui viendraient à les constater, devront sanctionner de telles situations "avec la plus extrême sévérité et la plus nécessaire rigueur" (point II-B-2). Le ministre demande aux fonctionnaires de recourir aux sanctions en vigueur dans la réglementation sociale et économique. (point III-2).

Là encore, l'imbrication de mesures conventionnelles illicites dans un tissu juridique dont la validité n'est par ailleurs pas contestée va permettre de favoriser leur mise en œuvre. L'État se propose d'appliquer les sanctions fixées en cas d'inapplication d'une disposition réglementaire lorsque l'employeur n'appliquera pas les dispositions propres à la convention.

Certes, il semble à première vue impossible de sanctionner un employeur qui ne respecterait pas les dispositions conventionnelles sur la durée maximale des temps de services, puisqu'elles sont illégales. Mais cet employeur, a fortiori, ne respecte pas non plus les textes réglementaires plus contraignants. De plus, l'article L. 153-1 CT permet de sanctionner pénalement le non respect de dispositions conventionnelles dérogatoires à la loi. L'administration sanctionnera donc sur cette base. Elle ne sanctionnera pas à l'inverse celui qui, appliquant les dispositions illégales de l'accord, fera fi des dispositions réglementaires.

34 - Nouveaux articles R. 241-9-1 et R. 241-9-2 CSS. 
Certaines affaires ayant abouti à la condamnation pénale de transporteurs peuvent être comprises en ce sens. Ces transporteurs n'avaient pas totalement appliqués l'accord "grands routiers". Plus précisément, les modalités d'application de cette convention, fixées par accord d'entreprise, n'étaient pas correctement appliquées. Les tribunaux ont alors fait un retour aux seules dispositions réglementaires pour sanctionner l'employeur ${ }^{35}$.

L'administration, qui était à l'origine de l'action publique engagée contre ces transporteurs, n'a pas interprété ces décisions de justice comme un désaveu de l'accord du 23 novembre 1994. Elle a au contraire considéré qu'il était normal de revenir à la législation de l'Etat dès lors que des transporteurs n'avaient pas loyalement appliqué l'accord ${ }^{36}$.

L'application des sanctions n'est donc pas uniforme. Elles dépend plus de la politique menée par l'État et définie par voie de circulaires que de l'irrespect des textes légaux auxquels sont attachées ces sanctions. Il reste que nous sommes ici fort proches d'un détournement de procédure sinon même de ce que, en droit privé, on dénomme une fraude à la loi. Sous couvert d'assurer sa mission d'application du droit, l'Etat met en œuvre une autre politique. Il dénie aux dispositions d'ordre public toute valeur impérative en ne les mettant en œuvre que de façon circonstancielle.

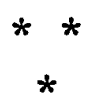

En dépit d'informations contradictoires, l'accord "grands routiers" ne semble être suivi que par un petit nombre de transporteurs, qui peinent d'ailleurs à le respecter ${ }^{37}$. Il n'a pas non plus permis, dans l'immédiat, une dynamique permettant une baisse globale de la durée du travail. Celle-ci reste toujours aussi

\footnotetext{
35 - Cf. notamment, Trib. de police de Pithivier, 26.2.1998 BTL ${ }^{\circ} 2743$ 9.3.1998, p. 171 et s. et BTL $n^{\circ}$ $2744,16.3 .1998$, p. 201.

36 - Cf. les propos de l'inspecteur général du travail et des transports (BTL n 27439.3 .1998 , p. 173 et BTL $n^{\circ} 2749$ 20.4.1998, p. 291) et ceux du Ministre des transports (BTL $n^{\circ} 2745$ 23.3.1998, p. 214).

37 - Cf. BTL n 2679 du 28.10.1996, p. 729 ; BTL n' 2684 du 2.12.1996, p. 822- ; BTL ${ }^{\circ} 2723$ du 13.10.1997, p. 688 et s. et BTL ${ }^{\circ} 2727$ du 10.11.1997, p. 772.

Il y aurait certainement beaucoup à dire des "statistiques" contradictoires qui sont publiées, concernant ce secteur : volonté des professionnels du transport de "brouiller les pistes", difficultés à obtenir des chiffres exacts du fait de l'absence de pratiques normalisées, caractère particulièrement hétérogène du secteur, obligeant à une rigueur méthodologique, qui n'a pas encore été atteinte ?
} 
importante ${ }^{38}$. Cet accord avait pourtant pour objectif de "coller" au temps réel de travail des conducteurs salariés puis d'en permettre une diminution progressive. L'accord exige cependant une comptabilisation précise de ces temps, ce qui est contraire à la pratique.

Cet accord n'est-il donc qu'un coup d'épée dans l'eau, et le récent arrêt de la Cour de cassation ne porte-t-il pas le "coup de grâce" à cette convention ${ }^{39}$ ? Toute discussion sur le caractère juridique de l'accord "grands routiers" n'est-elle pas quelque peu gratuite si l'on ne peut déceler aucun effet à celui-ci ? Des règles si mal appliquées ont-elles encore une quelconque positivité ? Mais dans ce cas, ce qui est vrai de l'accord l'est aussi de la réglementation étatique, tout aussi mal respectée, alors qu'elle a vocation à s'appliquer impérativement à tous. Pourtant, il ne viendrait à personne l'idÈe d'affirmer que cette réglementation n'a aucune réalité !

Quoique fort mal appliqué, l'accord du 23 novembre 1994 a le mérite de montrer que la vie du droit n'est pas forcément sous la complète dépendance de l'ordre juridique voulu par l'Etat. L'arrêt du 24 novembre 1998 semble certes indiquer le contraire. Cependant, cette décision intervient tardivement, presque quatre ans, jour pour jour, après la conclusion de l'accord. De plus, il se peut que l'accord "grands routiers" survive aux décisions de la Cour suprême, cela pour une raison très simple : cet accord tend à combler le "vide" juridique né de la très mauvaise application de la réglementation étatique.

En effet, l'inanité des règles fixées par le législateur n'est pas sans poser de délicates questions. Le problème n'est pas tant qu'il manquerait à ces normes toutes les caractéristiques d'une règle juridique mais plutôt que l'ordre juridique étatique ne serait pas exactement ce qu'il se donne à voir, soit des règles d'ordre public, d'application stricte ${ }^{40}$.

38 - 55,5 H. en moyenne hebdomadaire, en 1997 , et jusqu'à $61,5 \mathrm{H}$. pour les très grands routiers (plus de 3 nuits de découché par semaine) : enquête ministère du transport (Cf La vie du rail et des transports du 65-1998, p. 14); cf. également BTL n²749 20-4-1998, p. 291.

39 - Crim. 24-11-1998 B.T.L. ${ }^{\circ} 2783,18-1-1999$, p. 18. Une chose est certaine, l'Etat aura désormais le plus grand mal à sanctionner pénalement ceux qui, s'inscrivant avec réticence dans le cadre de l'accord du 23 novembre 1994, étaient poursuivis sur le fondement du non respect du Code du travail. L'employeur poursuivi pourra toujours rétorquer qu'il tendait à appliquer l'accord "grands routiers", sur les injonctions de l'administration du travail, que ladite administration l'ayant mal informé, il peut bénéficier, à titre d'excuse, de "l'erreur de droit".

40 - Sur l'ensemble de ces questions, cf. S. Carré : "L'état du droit dans le transport routier de marchandises : une réglementation en trompe l'ail" Droit et Société 1999, à paraître. 
Car ce que l'on peut reprocher à la législation de l'Etat, on ne peut le faire de l'accord "grands routiers". Conclu afin de mettre entre parenthèses une réglementation impérative, sa philosophie n'est évidemment pas d'avoir les mêmes exigences. La technique conventionnelle ne le permet d'ailleurs pas, spécialement sous l'angle de la pénalisation des comportements non respectueux des règles fixées ${ }^{41}$. La philosophie de l'accord est d'encadrer et de faire évoluer de manière souple et graduée les pratiques de la profession.

La commission de suivi de l'accord a manifestement une fonction pédagogique et persuasive. Il s'agit de faire pression. Les comités régionaux mis en place n'ont pas d'autres fonctions que d'inciter et d'impliquer les entreprises locales. Les fonctionnaires chargés de faire respecter cet accord prennent avant toute chose contact avec les chefs d'entreprises et prodiguent leurs conseils.

Les chartes cadres souscrites au niveau régional suivent la même démarche $^{42}$. Il s'agit, plus globalement, d'obtenir l'adhésion au "Contrat de progrès", dont les objectifs ont été progressivement repris dans l'accord "grands routiers", mais encore au cours des réformes législatives intervenues durant les annćcs 1990. Cette démarche, qui ne concerne donc pas que l'accord "grands routiers", mais vise aussi la réglementation étatique, montre combien le décalage est grand entre ce que cette législation paraît être et ce qu'elle est en vérité : en quoi est-il donc nécessaire d'engager des actions de sensibilisation au respect de règles impératives?

N'est-ce pas la notion d'ordre public qui est remise en cause si l'on considère qu'une règle impérative a pour fonction essentielle d'être strictement suivie ? Tout laisse à penser que le législateur ne s'est jamais véritablement donné les moyens d'atteindre cet objectif. Dans les années quatre-vingt, la faible cohérence des textes légaux existants et l'inaction de l'administration, ont de la sorte permis d'entretenir une réelle distance à la loi.

41 - Encore faut-il rappeler l'article L. 153-1 CT qui dispose que les règles conventionnelles dérogatoires à la loi ou aux règlements peuvent, en présence d'infractions à leurs dispositions, entraîner l'application des sanctions pénales prévues en cas de non respect des textes légaux auxquels elles dérogent. C'est donc simplement en présence de dispositions conventionnelles plus favorables que la loi ou des règlements qu'aucune sanction pénale ne peut-être prise à l'encontre de celui qui ne les respecte pas.

42 - Ainsi, celle des Pays de Loire, souscrite par l'État, les chambres consulaires, les chargeurs et les distributeurs et les organisations de transporteurs, le 10.6.1997. 
De façon similaire, les règles aujourd'hui posées semblent avoir une autre fonction que celle d'être immédiatement appliquées. Il s'agirait plutôt de mettre progressivement en branle une dynamique. Semblent en attester l'évolution dorénavant constante de la législation, un renforcement progressif des contrôles $^{43}$, l'existence d'une pression des organisations professionnelles sur les entreprises du secteur, la présence d'un calendrier au sein même de l'accord de novembre 1994. 